\title{
Revolución y contrarrevolución en la ciudad global: las expectativas frustradas por la globalización de nuestras ciudades
}

\begin{abstract}
A pessimistic analysis, even catastrophic, prevails in studies of global urbanization. For example, the Reports of the UNDP-Habitat or the Worldwatch Institute, or the articles and presentations in intellectual fora. This does not mean that the transformative and integrative potential of cities is not recognized. The positive discourse about citizens' liberties that the urban revolution, currently in process, makes possible forms part of our culture. These are the two sides of this reality. Many cities, European and American, have promoted regeneration of their central areas that, in many cases, deal with almost an entire municipality, with a revaluing of the compact, heterogenous city characterized by the quality of public space. However the urban developments, the metropolitan regions, multiply inequalities over the landscape, generating spaces that are physically fragmented and socially segregated. The current urban revolution is frustrating. The political, urbanistic and cultural challenge today is to 'make the city' in all regions characterized by urbanization.
\end{abstract}

Key words: urban revolution, globalized urbanism, 'make the city', public space, perverse effects

\section{Resumen}

El análisis pesimista, incluso catastrófico, prevalece en los estudios sobre la urbanización global. Por ejemplo, los Informes de PNUD-Habitat o del Worldwatch Institut. O los artículos e intervenciones en encuentros intelectuales. Lo cual no obsta que se reconozca el potencial transformador e integrador de las ciudades. El discurso positivo sobre las libertades ciudadanas que la revolución urbana en curso hace posible forma parte de nuestra cultura. Son dos caras de la realidad. Muchas ciudades, europeas y americanas, han promovido la renovación de sus áreas centrales, que, en muchos casos, abarca casi todo el municipio con una revalorización de la ciudad compacta y heterogénea, caracterizada por la calidad del espacio público. Pero los desarrollos urbanos, las regiones metropolitanas, multiplican las desigualdades en el territorio, generan espacios confusos, físicamente fragmentados y socialmente segregadores. La revolución urbana actual es frustrante. El desafío político, urbanístico y cultural es hoy "hacer ciudad" en el conjunto de las regiones caracterizadas por la urbanización.

Palabras clave: revolución urbana, urbanismo globalizado, hacer ciudad, espacio público, efectos perversos 


\section{Introducción}

$\mathrm{L}$ a reflexión inicial sobre el enfoque del artículo se produjo después de la celebración celebrado en Barcelona en el año 2004. El autor, junto con Mireia Belil, directora de los Diálogos del Forum, organizó a lo largo de dos semanas un conjunto de encuentros sobre lo que podríamos denominar "la aventura urbana de finales de siglo", en los que participaron varias decenas de expertos durante la primera semana y un centenar de dirigentes de movimientos sociales durante la segunda semana. Estos encuentros, especialmente los celebrados en la primera parte entre expertos, le confirmaron la sospecha de que era imprescindible realizar un análisis "dialéctico" del proceso urbano que pusiera en primer plano las contradicciones del mismo. Un proceso contradictorio que se reflejó en las intervenciones de todos los participantes, como se expone en la primera parte del artículo.

El Forum y los diálogos sobre la ciudad le dejaron un sentimiento también contradictorio. En un marco que era, a la vez, algo parecido a una culminación del exitoso modelo urbanístico de Barcelona y una prueba de sus limitaciones y de su incipiente degeneración, los diálogos tanto de los expertos como de los dirigentes sociales generaban satisfacción y malestar. La calidad de los participantes y el público numeroso, el valor del pensamiento crítico de los intelectuales y profesionales, la fuerza del pensamiento y de la acción de los dirigentes sociales, las conclusiones radicales que se desprendieron de los encuentros, todo ello generaba ilusión y satisfacción. Pero en aquel marco, en aquella imagen tan contradictoria del "modelo" barcelonés, en aquella extraña libertad para proclamar la crítica más radical sin que esta declaración tuviera la mayor trascenden-

\footnotetext{
Director del Programa Gestión de la Ciudad, Universitat Oberta de Catalunya. E-mail: jborjas@uoc.edu. El autor de este texto considera que debe explicitar que en la redacción de este artículo el coeficiente personal, es decir, la implicación subjetiva, está muy presente a lo largo de todo el escrito. Ante todo, desea agradecer muy sinceramente a los responsables de la Revista Eure, que le propusieron esta colaboración, proporcionándole el placer y el privilegio de estar presente en este número tan especial. Pero de esta implicación emocional ya hablaremos luego.
}

cia, la alegría no podía ser mucha. El autor tuvo una sensación parecida a la que probablemente le hubiera provocado la celebración de un seminario sobre marxismo y alienación o sobre precariedad del trabajo y marginalidad en los almacenes de El Corte Inglés. Como ven, las contradicciones estaban servidas.

Una primera versión de este artículo se hizo a modo de presentación de dos interesantes textos de David Harvey y de Neil Smith, ambos conferenciantes en los diálogos citados. Esta presentación fue la ocasión de redactar unas primeras reflexiones sobre "la revolución y la contrarrevolución urbanas". La propuesta de EURE de aportar un texto al número 100 de la revista me ha dado la ocasión de rehacer el texto inicial, casi un borrador, para ofrecer un artículo más extenso y completo.

Como el lector podrá comprobar, el artículo incluye una última parte que rompe el carácter teórico o, por lo menos, general del texto, para ofrecer un análisis muy sintético y crítico del urbanismo de Barcelona. De nuevo aparecen las contradicciones. En la realidad del proceso barcelonés y en la posición del autor. Desde los inicios del proceso democrático en los primeros años ' 80 hasta mediados los ' 90 , el autor estuvo directamente implicado en la gestión de la ciudad y ocupó diferentes responsabilidades de gobierno en la misma. Y debe confesar que, con mayor o menor fortuna, contribuyó a presentar una imagen "globalmente positiva" del urbanismo barcelonés. A lo largo de la década de los '90 se hace evidente que el indudable progreso de la ciudad en la calidad de su oferta iba aparejado de un conjunto de aspectos negativos y de déficits sociales y culturales. Y, progresivamente, adquirió conciencia de que si bien no era preciso pasar del blanco al negro, sí que convenía presentar las dos caras, las contradicciones, del desarrollo urbano barcelonés. El autor se siente vinculado a este desarrollo y continúa pensando que mantiene muchos aspectos positivos, pero, aun manteniendo una actitud de colaboración con las políticas públicas de la ciudad, considera necesario presentar un análisis crítico y, en la medida de lo posible, propuestas alternativas respecto a algunas tendencias y algunos proyectos.

Finalmente, y pido excusas por el carácter personal de este último comentario, quiero explicar 
la emoción que me produce tener la oportunidad de colaborar en este número emblemático de EURE.

Llegué a Chile por primera vez los primeros días de julio de 1973. Invitado por el Instituto, entonces llamado CIDU, para realizar en él una estadía de tres meses. Me incorporé al equipo de investigadores y el director de entonces, el entrañable amigo Guillermo Geisse, me pidió que elaborara un extenso trabajo que sirviera de introducción a un encuentro internacional que debía celebrarse en Santiago a partir del 18 de septiembre. A lo largo de dos meses y medio viví intensamente en el Instituto, en la ciudad, en el país. Y fui escribiendo el texto solicitado, sobre estructuras urbanas y movimientos sociales. Llegó el fatídico día 11, el texto no estaba terminado, pero la generosa experiencia que lideraba el presidente Allende sí.

El seminario fue suspendido obviamente, pero decidí terminar el texto. Las largas horas de encierro en el apartamento debido al toque de queda me permitieron completar algunas lecturas y terminar la redacción del documento. Debía ser publicado en la revista EURE, en el número 8; tampoco fue posible. Pero quedó escrito, como un acto de resistencia al golpe militar y como un acto de adhesión, afecto y solidaridad al Chile que amé. Un acto de lealtad y adhesión al CIDU, de admiración a la vitalidad popular que respiré en Santiago, de agradecimiento y cariño al compañerismo insobornable que me demostraron los amigos del Instituto, de la Universidad Católica y de otras instituciones y organizaciones de aquel Chile.

Mi estadía en Chile duró tres meses. A comienzos de octubre, como estaba previsto, regresé a Barcelona. Guillermo me había propuesto antes del 11 de septiembre que regresara pronto para una nueva estadía. Y deseaba hacerlo. No fue posible. Me fui con el texto destinado a EURE, que publicó primero el Departamento de Geografía de la Universidad Autónoma de Barcelona y luego formó parte de un pequeño libro que editó SIAP. No me separé de Chile, del Chile que quería, en el exilio, en la distancia. Y luego, a partir de 1983, regresé, y desde entonces Chile forma parte de mis tiempos y mis espacios de vida.

\section{Los urbanistas, los investigadores urbanos y la ciudad capitalista}

¿Quién teme a la ciudad futura? Es decir a la ciudad actual que anuncia el futuro próximo, el cual ya está marcado por las dinámicas en curso. David Harvey, uno de los pensadores más críticos sobre la ciudad actual titula uno de sus libros más recientes Espacios de esperanza (2005), y no es precisamente la esperanza lo que se desprende de su análisis. Saskia Sassen, la famosa autora de la optimista obra The global city (1991) que tuvo como efecto colateral que muchas ciudades más o menos grandes reclamaran un buen lugar en un ranking inicialmente limitado a tres, ha escrito posteriormente textos críticos, casi apocalípticos, denunciando el emergente "fascismo urbano" y anunciando la rebelión de las hordas marginales de las periferias. Los autores citados, como los que citaremos a continuación, han visitado Barcelona en los últimos dos o tres años, y sus exposiciones orales y sus comentarios informales en privado acentuaban su pesimismo crítico. Una actitud que especialmente caracteriza a reputados investigadores y planificadores norteamericanos. Como el ya citado Neil Smith (colega de Harvey en la City University de New York); Michael Cohen (Cohen, 1991; Cohen, Blair \& Tulchin, 1996), ex directivo del Banco Mundial; Mike Dear, autor de un libro ya clásico sobre el nuevo modelo urbano (Dear, 2002); o Tom Angotti (profesor de la City University New York, editor de Planner's Network; Angotti, 1993), los cuales presentaron un panorama muy crítico, algunos casi apocalíptico, de las ciudades de hoy, tanto en lo que se refiere a Norte América como al resto del mundo (en el marco del Forum mundial de las culturas, Barcelona, septiembre 2004). Y algo parecido ocurre con otros destacados intelectuales, y de perfil diverso, como Michael Sorkin (2005), Mike Davis (2004 y 2006), Peter Marcuse, Richard Sennett (2003), Edward Soja (2000), Arjun Appadurai (2001, 2002 y 2007) o Richard Ingersoll (1996), a los que hemos tenido oportunidad de escuchar y en algunos casos hemos podido debatir con ocasión de sus visitas. Una relativa excepción, con un discurso que enfatizaba las nuevas posibilidades que ofrece la revolución informacional fue otro ilustre visitante: William Mitchell, de MIT, el autor de E-topia, con posiciones similares a las de Manuel 
Castells. En cambio la sensata y prestigiosa Jane Jacobs, en la excelente entrevista que le hizo la revista Urbanisme de París (2005) poco antes de su fallecimiento, acentuaba considerablemente el diagnóstico crítico que se anunciaba en su obra clásica sobre las ciudades norteamericanas ${ }^{1}$. Todos ellos priorizaban la crítica, incluso la denuncia, y dejaban en un plano muy secundario las dinámicas esperanzadoras y las propuestas correctoras. Lo mismo que el arquitecto israelí Eyal Weizman. La coincidencia entre autores marcadamente "radicales" o marxistas como Harvey, Smith, Angotti o Davis con otros de talante más "liberal" (en la cultura política norteamericana equivale a socialdemócrata) como Cohen, Sassen, Dear o Sorkin es significativa. Nuestra época replantea la razón de ser de la ciudad, las dinámicas disgregadoras son muy fuertes y las incertidumbres sobre su futuro crecientes. La coincidencia y la influencia global de los autores citados no permiten considerar que estas visiones están demasiado influenciadas por la lógica desazón provocada por la lamentable experiencia de vivir bajo la presidencia de Bush. $\mathrm{O}$ atribuir estas posiciones, propias de intelectuales críticos, a que se trata de analistas de la realidad pero no comprometidos con la gestión de la ciudad. Los autores citados son o han sido también profesionales que han estado al frente de programas de desarrollo o de proyectos urbanos, o han trabajado para instituciones políticas o han asesorado movimientos sociales con propuestas alternativas; no son críticos instalados en torres de marfil.

Hemos destacado las intervenciones de los expertos norteamericanos por el evidente contraste entre su pertenencia al país más potente y poderoso del planeta y el radicalismo de su crítica. Si consideramos a continuación las intervenciones públicas y privadas de intelectuales y expertos urbanos europeos o latinoamericanos, de perfil igualmente independiente y crítico, nos llama la atención que su discurso contiene elementos más "positivos". En sus obras recientes y en sus intervenciones en los encuentros internacionales citan experiencias constructivas o de planeamiento, propuestas y

La autora de The death and life of great american cities (1961), publicó en 2004 un interesante libro de memorias con el título Dark age ahead. programas interesantes y manifiestan un cierto optimismo sobre el futuro de la ciudad.

Evidentemente estos profesionales y analistas de la ciudad europeos y latinoamericanos, siempre de esta muestra arbitraria que hemos seleccionado, a los que hemos leído, visto y escuchado en directo en los últimos tres años, también expresaron posiciones críticas, pero el discurso era más propositivo. Nos referimos a personalidades tan distintas entre sí como los portugueses Nuno Portas y Boaventura de Souza Santos, los brasileños Fernando Enrique Cardoso, Jorge Wilheim, Jaime Lerner y Raquel Rolnik, los mexicanos Enrique Ortiz, Alicia Ziccardi y Alejandra Moreno Toscano, los chilenos Alfredo Rodríguez, Ana Sugranyes, María Elena Ducci y Carlos de Mattos, el ecuatoriano Fernando Carrión, los argentinos Eduardo Reese, Fredy Garay, Marta Aguilar y Carlos Reboratti (aunque en el caso argentino destaca también el discurso radicalmente crítico de Graciela Silvestri o de Adrián Gorelik), los franceses François Ascher, Jean Louis Cohen, Olivier Mongin, Ariella Masboungi, Patrick Viveret, Michel Marcus, François Barré, Sophie Body-Gendrot, los italianos Giusseppe Campos Venuti, Laura Balbo, Francesco Indovina, Mauricio Marcelloni, Bruno Gabrielli, Franco Corsico, los redactores de revistas de matriz italiana como Domus, Area o Giornali delli architetti o las francesas Urbanisme y Projet Urbain, los británicos Peter Hall, Richard Rogers, Tim Marshall, los catalanes Manuel Herce, Josep $\mathrm{M}^{\mathrm{a}}$ Montaner, Francesc Muñoz, Oriol Bohigas, Manuel Solà Morales, o los madrileños Jesús Leal, Eduardo Leira, Eduardo Mangada, Luis Fernández Galiano, o el ya citado y difícil de ubicar Manuel Castells. Una lista no exhaustiva, pues no podemos citar a todos los que hemos podido escuchar con interés en el marco de eventos sobre temas urbanos ${ }^{2}$.

No estamos en condiciones de aportar una explicación suficientemente fundada a este contraste entre unos y otros. Probablemente existen varias explicaciones. Es posible que alguno de los expertos norteamericanos nos diga que no es un

2 No citamos bibliografía de todos estos autores para no hacer más pesado el texto. En Borja (2005), citamos obras de la mayoría de ellos. 
pesimista, sino "un optimista informado". Y añadiría que el rol imperial que ha asumido Estados Unidos en el mundo les "obliga" a tener en cuenta el conjunto del planeta y por lo tanto integran en su análisis el mundo menos desarrollado y sus actuales procesos de urbanización caótica, insostenible e ingobernable. A nosotros, los representantes de la "vieja Europa" nos podrían reprochar no apercibirnos que nuestras ciudades consolidadas, históricas, de perfil cultural propio, son fantasmas del tiempo pasado, destinadas a museo o parque temático para turistas o a última residencia para la cuarta edad más o menos acomodada del mundo. Y podrían añadir: miren a su alrededor, las nuevas formas de urbanización de ustedes no son diferentes de las nuestras, simplemente están por ahora menos desarrolladas.

Quizás podemos apuntar otro tipo de explicación, complementaria, que se refiere a las condiciones de producción e intercambio de la producción del conocimiento. La política del actual gobierno norteamericano personificada en la figura de su presidente ha generado mucho rechazo en Europa. Y como consecuencia de ello las iniciativas culturales europeas han privilegiado las relaciones con intelectuales, académicos o profesionales, de marcada e indiscutible posición crítica y, por lo tanto, alejados de los ámbitos de poder. Así ha sucedido con los nombres citados al inicio de este artículo, que han sido objeto de invitaciones (a veces repetidas, incluso frecuentes) por parte de entes culturales, en este caso de Barcelona, vinculados al poder político.

En Europa la simbiosis entre intelectuales y profesionales críticos y el poder político es frecuente, o lo ha sido, y en temas urbanos no hay una separación radical entre el mundo académico y cultural y la inserción en redes articuladas con las administraciones públicas. En América Latina los procesos democratizadores de las dos últimas décadas también han creado conexiones entre el poder político y los sectores intelectuales y profesionales procedentes de la cultura crítica de los años ' 60 y '70. Lo cual ha llevado incluso a destacados pensadores radicales a integrarse en estructuras de gobierno, tanto a nivel local como estatal. El resultado es visible: la mayoría de los europeos y latinoamericanos citados son a la vez representantes del pensamiento crítico o radical y son, o han sido, políticos o profesionales con responsabilidades de gestión pública o de diseño o ejecución de programas y proyectos destinados a las ciudades.

No es un detalle sin importancia indicar "desde dónde se habla", desde dónde se investiga, se planifica o se escribe. Estamos demasiado acostumbrados a considerar un estudio o un artículo "en sî”, como si fuera indiferente la posición social o profesional de su autor, o suponiendo que implícitamente el lector ya conocerá y establecerá por su cuenta la adecuación entre esta posición y el texto, es decir procurará adivinar el punto de vista desde donde se escribe. Justo porque esta adecuación no se debe considerar como automática hemos iniciado este documento situando a los autores citados, que unos más que otros, han influido en el firmante de este texto.

La mayoría de autores nos proponen claves interpretativas sobre las ciudades en la globalización, en unos casos mediante exposiciones teóricas sobre la ciudad actual y en otros deduciendo consecuencias para su posterior intervención en ciudades o territorios concretos. El análisis de la relación entre globalización y urbanismo les lleva a constatar la profunda crisis de las políticas locales de "reproducción social" (vivienda, educación, servicios sociales, etc.), que han sido históricamente propias de los gobiernos municipales o regionales. Los nuevos espacios locales, la ciudad extensa o el espacio urbano-regional, se han convertido por otra parte en territorios de organización de la "producción social" (conjunto de factores que intervienen en el proceso económico de producción de bienes y servicios), pero de gobernabilidades débiles y fragmentadas. Lo cual conlleva a que se agudicen las contradicciones y se acentúen las políticas "revanchistas" o represivas (Gambrill, 2002). Autores tan distintos como Smith, Sassen, Harvey o Angotti, a partir de su conocimiento de las ciudades norteamericanas, denuncian la emergencia de un "fascismo urbano" como ya hemos citado al inicio de este texto. En resumen el conflicto social (o la nueva lucha de clases) se ha desplazado, relativamente, del ámbito del Estado-nación y del lugar de trabajo a los territorios locales y al nexo entre lo local y lo global (Borja y Castells, 1997; ver también Castells, 1997 y Borja, 2003). 
En el caso europeo los autores a los que hemos hecho referencia se mueven entre dos polos. En un caso enfatizan la adecuación de la ciudad a la globalización, su inserción en redes macrorregionales (continentales, mundiales), el ganar posiciones competitivas y conseguir funciones nodales y atraer flujos. Es la ideología naturalizadora del actual capitalismo que sirve de señuelo para orientar las políticas urbanas y que se traduce en la arquitectura de autor, la oferta de áreas para la nueva economía, la gentrificación (o museificación) de la ciudad consolidada, la mercantilización del valor simbólico del patrimonio, el miedo justificador de los barrios cerrados, el crecimiento periférico por piezas y funciones especializadas, etc. El otro polo es el de la resistencia a la globalización, o a sus efectos perversos, que se manifiesta cuando se comprueba: la dificultad de promover un desarrollo sometido a lo global y que sea compatible con la cohesión social y la sostenibilidad ambiental, la creciente marginación de poblaciones sacrificadas en aras de la competitividad global, la banalización y pérdida de identidad de los territorios, etc. Reaparece entonces el discurso sobre la ciudad, el espacio público, la calle, la mixtura social, el perfil identitario y el patrimonio como memoria urbana $^{3}$.

En el caso de los autores latinoamericanos la necesidad de integrar ambos polos es aun más urgente. Por una parte las ciudades deben mejorar su inserción en flujos globales para recuperar la brecha tecnológica, financiera y económica del último tercio del siglo XX. Por otra parte los déficits sociales, culturales y de gobernabilidad democrática deben reducirse si se quiere evitar una crisis urbana generalizada.

Es decir, que nos encontramos ante unas realidades urbanas extremadamente contradictorias y unas dinámicas tanto privadas como públicas que se instalan en estas olas opuestas y producen discursos ambivalentes. Los analistas y planificadores, por ahora, evaden la contradicción, y cuando no construyen un discurso inevitablemente genérico

Veáse la reciente exposición y catálogo sobre "La calle nos pertenece" comisariada por François Ascher y organizada por el Instituto de la Movilidad urbana en París (2007) y que próximamente llegará a América Latina (Buenos Aires, 2008). e inoperante (como ocurre frecuentemente en la moda del planeamiento estratégico) toman partido por uno de los polos. El problema es que por ahora no parece posible que un polo elimine al otro.

Para terminar esta primera parte introductoria nos referiremos a dos informes recientes sobre la ciudad en el mundo actual elaborados por organismos de las Naciones Unidas. Uno es del Fondo de Población (UNFPA): Estado de la población mundial 2007. Liberar el potencial de crecimiento urbano. El otro es de ONU - Habitat: State of the World's Cities 2006-07. A los cuales se puede añadir el último Informe de Worldwatch Institute: State of the World 2007. Our Urban Future. En primer lugar es significativa la coincidencia. Estos tres informes, todos ellos referidos al estado del mundo, coinciden en un enfoque exclusivamente urbano. En segundo lugar es interesante que todos ellos no se limitan, como ha sido tan frecuente en el pasado, a presentar la realidad urbana global como catastrófica o por lo menos muy preocupante, también exponen el potencial resolutivo de los problemas que se encuentra en las ciudades (como dice Jaime Lerner en su intervención en el Forum Mundial de las Culturas, Barcelona 2004, "la ciudad no es problema, es solución”). Es significativo el subtítulo del Informe de UNFPA: el potencial del crecimiento urbano. Y tanto este Informe, como el de Habitat y el de Worldwatch, indican una gran diversidad de propuestas razonables para abordar con éxito los problemas generados por la urbanización del mundo. En tercer lugar, sin embargo, las soluciones parecen depender de la voluntad de las administraciones públicas y de la movilización de la ciudadanía, y no se entiende muy bien por qué no se aplican y se generalizan.

Estos informes, todos ellos de gran interés, nos confirman las contradicciones tanto del desarrollo urbano como de las políticas y de los comportamientos sociales vinculados a la urbanización. En el texto que sigue analizamos estas contradicciones, o por lo menos las que nos parecen más significativas. Nuestra hipótesis de partida es que la emergencia de la ciudad del siglo XXI es espectacularmente dialéctica, y en ella lo bueno y lo malo, integración y marginalidad, cohesión social y desigualdad creciente, desarrollo sostenible y dinámicas insostenibles, productividad compe- 
titiva y enclaves excluyentes, democratización de la gestión urbana y crisis de gobernabilidad de las regiones urbanizadas, globalización y localismo, etc., están en conflicto permanente.

Hemos tenido en cuenta las contribuciones de los autores citados y otros que han analizado las contradicciones de la ciudad post-industrial ${ }^{4}$. El texto que sigue expone brevemente las características y contradicciones de la "revolución urbana" de nuestra época. En segundo lugar las respuestas del urbanismo, la revalorización de la ciudad y las dinámicas fragmentadoras y segregadoras que se dan en ella. Y finalmente el debate sobre los modelos de ciudad en curso y su relación con el derecho a la ciudad propio de nuestra cultura democrática ${ }^{5}$.

\section{Revolución urbana y urbanismo}

\section{Sobre el uso de los términos revolución y contrarrevolución en la ciudad de la globalización}

Utilizamos los términos de revolución y contrarrevolución no solamente por la historia que conlleva consigo el concepto de revolución urbana y su renovación a lo largo del tiempo, como se comprueba en la literatura sobre el auge de las ciudades metropolitanas a lo largo del siglo XX y más recientemente sobre la "explosión de la ciudad" (ver el catálogo de la exposición realizada en Lisboa, Venecia y Barcelona que con este título dirigieron Nuno Portas, Francesco Indovina y Antonio Font) o el ya clásico concepto de metapolis acuñado por François Ascher. También lo usamos en un sentido más general que corresponde al hilo interpretativo de nuestro texto.

Las revoluciones, sean políticas, sociales, económicas, científicas, culturales o tecnológicas

\footnotetext{
4 Algunos títulos significativos son Ascher (2003), Castells (2000; 2001) y Amendola (2001).

El derecho a la ciudad, concepto que planteó Lefebvre en los años 60 , ha sido desarrollado muy recientemente por los movimientos sociales urbanos a nivel internacional, como la red liderada por Habitat International Coalition que ha elaborado una propuesta de Carta por el Derecho a la Ciudad. En la conferencia de Habitat de Estambul, en 1996, aún no se planteó, pero sí en el Foro Urbano Mundial de Habitat, en Barcelona el año 2004). Ver también Borja (2005) y Fundación Alternativas (2004).
}

generan procesos (o por lo menos expectativas) que para simplificar podemos calificar de "democráticos" o socializadores del progreso. En el caso de la revolución urbana de nuestra época, ampliamente descrita, se enfatiza la mayor autonomía de los individuos, la diversidad de ofertas (de empleo, formación, ocio, cultura, etc.) que se encuentran en los extensos espacios urbano-regionales, las nuevas posibilidades de participación en las políticas públicas de las instituciones de proximidad y a partir de la socialización de las nuevas tecnologías, las mayores posibilidades de elegir residencia, actividad o tipo de movilidad, etc.

Sin embargo, nunca la segregación social en el espacio había sido tan grande, crecen las desigualdades de ingresos y de acceso real a las ofertas urbanas entre la población, colectivos vulnerables o más débiles pueden vivir en la marginación de guetos o periferias (ancianos, niños, inmigrantes, etc.), los tiempos sumados de trabajo y transporte aumentan, la autonomía individual puede derivar en soledad e insolidaridad, la incertidumbre sobre el futuro genera ansiedad, se pierden o debilitan identidades y referencias, hay crisis de representación política y opacidad de las instituciones que actúan en el territorio, etc. Es decir las esperanzas generadas por la revolución urbana se frustran y el malestar urbano es una dimensión contradictoria de la vida urbana actual.

Estos efectos perversos de la revolución urbana no son una fatalidad sino que resultan de un conjunto de mecanismos económicos, de comportamientos sociales y de políticas públicas como son: el carácter sobredeterminante de la renta urbana en la definición de usos del territorio, el consiguiente carácter de "ahorro que han adquirido las inversiones en suelo o en vivienda, las alianzas "impías" entre promotores y autoridades locales, el afán de distinción y de separación de importantes sectores medios y altos, los miedos múltiples y acumulativos que actúan sobre una población de cohesión débil, la fragmentación de los territorios urbanos extensos y difusos, la homogeneización de pautas culturales en los que la "imitación global" se convierte en obstáculo a la integración local, etc. Todo lo cual inidica que vivimos no solo tiempos de revolución, también son tiempos de contrarrevolución, urbana obviamente. 


\section{La revolución urbana: dimensiones sociales y territoriales.}

El término "revolución urbana”, que usó Gordon Childe en su obra clásica sobre la Antigüedad, ha sido recientemente recuperado por Ascher (2003). La revolución urbana no es la traducción directa de la globalización en el territorio pero sí que viene causada por un conjunto de factores tecnológicos, económicos, políticos, sociales y culturales que también se vinculan a la globalización, concepto por otra parte suficientemente confuso, lo que permite que sirva un poco para todo ${ }^{6}$.

a) La informatización (por ejemplo la difusión de las redes telemáticas (Internet, telefonía móvil) ha modificado las relaciones espaciotiempo y permite desarrollar actividades diversas (profesionales, de ocio o cultura, de educación, de consumo) sin depender de una localización rígida. Si a ello se une la generalización de las formas modernas de comunicación como auto privado y las redes regionales de transporte, es fácil deducir que la ciudad hoy ya no es lo que era.

b) Los nuevos territorios urbanos ya no se reducen a la ciudad central y su entorno más o menos aglomerado, lo que se llamó el "área metropolitana”, es decir el modelo de ciudad de la sociedad industrial. El territorio urbano-regional es discontinuo, mezcla de zonas compactas con otras difusas, de centralidades diversas y áreas marginales, de espacios urbanizados $\mathrm{y}$ otros preservados o expectantes. Una ciudad de ciudades en su versión optimista o una combinación perversa entre enclaves globalizados de excelencia y fragmentos urbanos de bajo perfil ciudadano.

c) El capital dominante es hoy financiero más que productivo, nómada más que sedentario. Las decisiones se han "externalizado" del territorio, el cual se ha vulnerabilizado al tiempo que ha entrado en la carrera competitiva para atraer inversiones, actividades emblemáticas, turistas, etc. El capital fijo,

Usamos el término globalización solamente en relación a sus impactos, reales o supuestos, sobre los territorios urbanos. dependiente del entramado económico local, se resquebraja, y las infraestructuras que soportan la nueva economía corren el riesgo de ser de uso efímero y de relevancia únicamente global pero no para el territorio en el que están insertas.

d) El ámbito local-regional ha sido históricamente el de la reproducción social (educación, sanidad, vivienda, etc.), hoy afectado por la crisis del welfare state (o por su carácter inconcluso) al mismo tiempo que las demandas se multiplican (formación continuada, envejecimiento, reducción del tamaño del núcleo familiar, colectivos pobres o marginales, etc.). Los poderes locales $\mathrm{y}$ regionales deben reorientar sus funciones hacia la "producción social" puesto que la "competitividad" del territorio corresponde a esta escala más que a la del "estado-nación”. Pero no disponen de las competencias y recursos para ello (Smith, 2005).

e) La sociedad urbana se ha hecho más compleja, más individualizada y más heterogénea. Las grandes clases sociales de la época industrial se han fragmentado, los grupos sociales se definen en función de criterios múltiples (territoriales, culturales, etc., además de su relación con la producción), la autonomía del individuo se ha multiplicado. Los comportamientos urbanos se han diversificado (en los tiempos, movilidades, relaciones sociales, etc.) y por lo tanto también las demandas. Las políticas urbanas hoy no pueden ser simplemente de "oferta" masiva dirigida a grandes colectivos supuestamente homogéneos.

f) Pero, paradoja: al mismo tiempo que individuos y ciudades apuestan por la distinción y la diferencia las pautas culturales se globalizan y se homogeneizan (Harvey, 2005). Arquitecturas y formas de consumo, informaciones y comportamientos de ocio, lenguas (las variantes del pseudoinglés) y vestimentas, se banalizan y pierden sus elementos distintivos cualificantes. La carrera hacia la competitividad mediante la distinción lleva a la no-competividad mediante la banalización. 
g) La gobernabilidad de los territorios urbanoregionales se convierte en un difícil desafío. Especialmente difícil debido a los factores citados que ahora resumimos:

i. La multidimensionalidad del territorio urbano-regional (centros, periferias, red incompleta de geometría variable de ciudades medias y pequeñas, urbanización difusa, enclaves y hábitat marginal, etc.).

ii. Las potentes dinámicas privadas de ocupación de suelo. Por ejemplo, en Barcelona - región metropolitana, en los últimos 25 años la urbanización del suelo se ha multiplicado por dos, siendo la población estable?.

iii. La nueva complejidad de la sociedad urbana y la diversidad de sus demandas y de sus comportamientos (movilidad, doble residencia...).

iv. La fragmentación de los poderes locales (entre 150 y 200 municipios en la región metropolitana de Barcelona, más comarcas, mancomunidades, entidades metropolitanas, Diputación, Generalitat, ministerios) que cooperan y se solapan, compiten, se estorban...

v. La fuerza económica y a veces legal de las iniciativas privadas o de entes públicos sectoriales a la hora de definir o

Se presentan algunos datos para ilustrar estas dinámicas de ocupación del suelo en las regiones urbanas de Barcelona y Madrid. Para el caso de la Región Metropolitana de Barcelona, el crecimiento de la superficie urbanizada entre 1972 y 1999 fue del 185\%, con un mayor crecimiento en la segunda corona, tal y como vemos en el trabajo de Manuel Herce en Borja y Muxí (2004). También entre 1987 y 2001 para el $78 \%$ de los municipios de la provincia de Barcelona más de la mitad de la producción de la vivienda había sido de tipología aislada o adosada. También cabe destacar que son los 137 municipios pequeños de la provincia (de 1.000 a 10.000 habitantes) los que han producido más del $80 \%$ de vivienda unifamiliar (Muñoz, 2004). Para el caso de la región urbana de Madrid, entre 1957 y 1999, el suelo urbanizado se quintuplica (de 10.700 a 49.000 hectáreass), mientras la población se duplica (2,3 a 4,7 millones). Datos que constatan la enorme expansión del territorio urbanizado en Madrid (Borja y Muxí, 2004). modificar grandes proyectos sectoriales sobre el territorio. La gobernabilidad de estos territorios exige una capacidad de innovación política que el marco institucional obstaculiza y que la deficiente representatividad de los partidos, convertidos en maquinarias electorales para ocupar posiciones en las instituciones, difícilmente supera (Borja, en prensa).

Las ciudades ante los efectos más o menos perversos de la globalización: entre la sumisión y la resistencia.

El análisis según una matriz explicativa de una sola dirección es más gratificante, te aseguras un sector de público partidario de tu discurso, y si otro sector lo critica, pues muy bien, es sabido que lo importante es que hablen de uno, y si hablan mal mejor, la gente se fija más. Pero, con el riesgo de no complacer ni a tirios ni a troyanos, me parece inevitable presentar un análisis que pone de relieve las ambivalencias más que las bondades o maldades de los procesos y de los discursos urbanos actuales. Y las conclusiones serán irremediablemente abiertas. Lo siento, el pensamiento dialéctico no produce automáticamente la síntesis superadora.

En el plano económico el discurso globalizador tuvo un arranque arrollador. La presentación de las ciudades como lugares nodales (Castells, 1997; Sassen, 1991), las nuevas oportunidades de los territorios (argumento apoyado en emergencias y reconversiones exitosas) y la prioridad al posicionamiento en las redes globales y en consecuencia a su proyección exterior han sido elementos clave de la construcción del vademécum de la buena política urbana. El plan estratégico a su vez ha sido la herramienta operativa (o ha pretendido serlo) de las ciudades aspirantes a triunfar en el mundo global mediante el discurso "hipercompetitivo". Un tipo de plan no normativo, que favorece tanto una concertación de cúpulas políticas con cúpulas económicas como un amplio proceso participativo. Y que puede convertirse en un proyecto político transformador de la ciudad o derivar en una cortina de humo llena de buenas intenciones, sin otra función que legitimar las prácticas del poder. 
Ha habido reacciones sociales y políticas críticas, que han denunciado, desde un plano ideológico, cómo la aceptación de la prioridad a la "competitividad" significaba aceptar casi siempre una posición de dependencia respecto a los mecanismos excluyentes que dominan la vida financiera y comercial internacional y que en la práctica acentuaban las desigualdades y la dualización social y territorial de la ciudad. Estas reacciones apuestan por planes de carácter más proteccionista, alternativos, que a su manera pueden ser modernizadores (por ejemplo la "nueva cultura del agua", reutilizadores del capital fijo y generadores de empleo. En bastantes casos la oposición a la adaptación acrítica a la globalización se fundamentó también en la revalorización de los elementos identitarios del territorio y en la defensa de la calidad de vida y de un desarrollo sostenible.

La síntesis teóricamente posible es cuadrar la ecuación competitividad, cohesión social, sostenibilidad, gobernabilidad y participación. No es evidente, y aun no se ha descubierto la piedra filosofal para ello, por lo menos rebus sic stantibus.

Es significativa la importancia creciente que adquieren los factores culturales en la orientación de los procesos urbanos actuales. Por una parte la conciencia de las desigualdades sociales, el afán de distinción, el miedo a los otros y el refugio en vida privada son muy funcionales al modelo del urbanismo globalizado, que describimos más adelante. Por otra surge una contestación cultural a los impactos de la globalización y de la economía de mercado sobre el territorio.

La defensa del patrimonio construido del paisaje, de la población y sus habilidades, de las relaciones sociales consolidadas por el tiempo, de la lengua y de las culturas específicas y la valorización de la animación urbana (por ejemplo de la calle y el espacio público como elementos fundamentales de la ciudad), todo ello cuenta cada vez más en la resistencia a los proyectos privados o públicos con fuertes impactos sobre el territorio. El rechazo a la homogeneización cultural que conlleva la

Nueva cultura del agua, concepto reivindicativo y alternativo forjado por el movimiento social de oposición al Plan Hidrológico Nacional y derogado por el actual gobierno de España. globalización también forma parte de los actuales procesos urbanos.

La cuestión es si estas resistencias son simplemente una oposición legítima pero conservadora o por el contrario pueden ser una palanca de desarrollo autocentrado en nuestro mundo global. En todo caso las anteriores reflexiones pueden matizar algunas de las posiciones más críticas que hemos citado al inicio de este texto.

\section{Urbanismo globalizado versus urbanismo ciudadano}

Un debate posible puede ser sobre los modelos de desarrollo urbano. Se construye un modelo abstracto, por ejemplo el "urbanismo ciudadano" que podemos contraponer al "urbanismo globalizado". Son modelos que con frecuencia se usan de forma maniquea, pero que indudablemente tienen una útil capacidad heurística. Por ejemplo Castells propone analizar el caso barcelonés mediante la oposición entre el modelo 1 (urbanismo ciudadano) y el modelo 2 (urbanismo globalizado). El arquitecto y teórico Josep $\mathrm{M}^{\mathrm{a}}$ Montaner ha analizado el urbanismo barcelonés y ha llegado a conclusiones similares (Montaner en Borja y Muxí, 2004). Es evidente que existen unas dinámicas territoriales empujadas por la globalización en un marco imperfecto de economía de mercado dominado por los que disponen de "rentas monopólicas" (para usar el lenguaje de Harvey). Pero también lo es que hay dinámicas de signo contrario o que modifican los efectos de las primeras.

El modelo de desarrollo urbano característico de la era de la globalización es el de la "urbanización difusa y discontinua" mediante "productos urbanos" constitutivos de enclaves o parques temáticos mercantilizados ${ }^{9}$ y áreas degradadas o marginales (Muxí, 2004). Una urbanización de suelo regional que puede darse sin crecimiento económico, en América Latina por ejemplo, o sin

Un indicador de la fuerza de esta tendencia es la reconversión de facto de entes públicos o parapúblicos (con participación de actores privados) creados para promover actividades industriales (y de servicios generadoras de empleo en las áreas urbanas en crisis), en agencias de promoción de suelo en áreas expectantes (no urbanizadas, o muy poco, en espera especulativa) y enclaves de ocio, comerciales, empresariales, tecnológicos o complejos residenciales de nivel alto. 
crecimiento demográfico como en Europa (Davis, 2006; Borja y Muxí, 2004). Es la urbanización que genera "espacios lacónicos" punteados por shopping malls y gasolineras (Ingersoll, 1996), de las "áreas de excelencia” (Castells y Hall, 1994) (parques empresariales o tecnológicos, barrios cerrados exclusivos), red de autopistas y estratificación social en función de la distancia-tiempo a los lugares de centralidad $^{10}$.

Sin embargo hay dinámicas de sentido contrario que encuentran también su expresión en el urbanismo actual. No tanto en el new urbanism ${ }^{11}$ que crea sucedáneos de "ciudad europea compacta" sino en el urbanismo "ciudadano" presente en grados diversos en las políticas de bastantes ciudades europeas y americanas. Es el urbanismo del "espacio público" y de la ciudad densa, de construcción de centralidades (Busquets, 2004; Ajuntament de Barcelona, 1988) ${ }^{12}$, de mixtura social y funcional ${ }^{13}$. La ciudad de Barcelona ha sido considerada casi como emblema o portavoz de este urbanismo, lo cual seguramente es excesivo, puesto que este modelo ha orientado muchas de las políticas públicas urbanas de la ciudad europea ${ }^{14}$. En la realidad es frecuente que ambas tendencias se mezclen y confronten en la misma ciudad ${ }^{15}$. Los dos "modelos" actúan casi siempre a la vez, o más exactamente, ayudan a interpretar ambos las políticas urbanas y el desarrollo contradictorio de la ciudad.

10 La planificación e inversión pública en infraestructuras de comunicación (viaria especialmente) es utilizada muchas veces para generar áreas con fuerte potencial especulativo (ver Herce en Borja y Muxí, 2004)

11 Ver Congress for New urbanism Basis en www.cnu. org/newurbanism.html. También Duany et. al (2000).

12 Sobre el modelo urbanístico de Barcelona ver especialmente Bohigas (1985).

13 Sobre la mixtura social, ver especialmente la interesante tentativa francesa, normativizada en la Ley de solidaridad y renovación urbana de 1999.

14 Ver el catálogo de la exposición La reconquista de Europa 1980-1999, obra de García Espuche y de Navas (1999). En esta obra se analizan los principales proyectos urbanos de las ciudades europeas a partir del protagonismo del espacio público. celona.
$\mathrm{La}$ arquitectura banalizada y estandarizada caracteriza al urbanismo "globalizado", lo mismo que el uso y el abuso de las arquitecturas ostentosas y "no reproducibles" (Baudrillard y Nouvel, 2000) ${ }^{16}$ para marcar simbólicamente las zonas de excelencia. El urbanismo "ciudadano" apuesta por el perfil identitario de lo urbano, atendiendo a la morfología del lugar, a la calidad del entorno y a la integración de los elementos arquitectónicos excepcionales o emblemáticos. Ambas tendencias pueden encontrarse en el mismo período y en las mismas ciudades pero conviene conocer qué fuerzas y qué actores empujan cada una y cuál tiende a imponer su lógica.

El efecto "político" de esta confrontación de modelos es incierto, aunque no cabe duda que con independencia de las voluntades políticas locales en el marco de la economía globalizada capitalista, de la propiedad privada del suelo y de la mercantilización de la vivienda, la tendencia dominante es la "urbanización difusa" y la producción de enclaves o parques temáticos de ocio. Es el urbanismo de la privatización, de la distinción y del miedo (Borja, 2005a; 2005b; 2006). En algunos países europeos se han implementado políticas urbanas de signo ciudadano, como en Gran Bretaña (Rogers, 2001) y en Francia (Masboungi, 19941997) pero en el mejor de los casos se obtienen resultados contradictorios, es decir un poco de todo. En Francia la hegemonía cultural del projet urbain que ha orientado el excelente urbanismo de diversas ciudades en los últimos veinte años no ha impedido la urbanización difusa, creciente y banal de una parte importante del territorio ${ }^{17}$.

Aunque siguiendo el razonamiento de Harvey se puede interpretar que el resultado final es muy funcional al urbanismo de la globalización, puesto que la competitividad entre los territorios requiere estos "lugares nodales de cualidad" que son las ciudades vivas, con espacios públicos animados y ofertas culturales y comerciales diversas, con entornos agradables y seguros, donde se concentra

16 Ver crítica al texto de Baudrillard y Nouvel (2000) en Silvestre (2002). Una crítica más general a la arquitectura no reproducible en Bohigas (2004).

$17 \operatorname{Kempf}(2005,14$ de abril), citando el informe Lepeltier, Ministro de Ecología. 
el terciario de excelencia y el ocio atractivo para los visitantes (Harvey 2005). Los residentes son los extras de la película.

El efecto directamente político de esta confrontación (desigual) de tendencias es el que nos plantea una incertidumbre sobre el devenir de la democracia en el territorio. Por una parte hay un resurgir del ámbito político urbano-regional. Se habla incluso, a favor o en contra, de las nuevas "ciudades-estado". En las regiones metropolitanas, como hemos visto al principio, se plantean problemas de gobernabilidad. Pero también son, o pueden ser, ámbitos de innovación política, como apuntan algunos procesos de descentralización, de contractualización interinstitucional, de gestión cívica o participativa, de concertación público-privada, de experimentación de democracia deliberativa y de e-gobernabilidad (electrónica o virtual).

Sin embargo las crecientes desigualdades en el territorio, la división cada vez más manifiesta entre "incluidos y excluidos" que caracteriza por ahora más a la ciudad americana que a la europea, pero también presente entre nosotros, pueden dar lugar a una "lucha de clases en el territorio" o una "conflictividad asimétrica" ${ }^{18}$ de difícil gestión en la fragmentada democracia local. La agudización de los conflictos entre colectivos sociales segregados puede desembocar en el "fascismo urbano" como recientemente anunciaba la citada Sassen $(2004)^{19}$. La conocida autora de "la ciudad global" advierte que en muchas ciudades la rebelión social, que tenderá a expresarse en las periferias marginadas, tendrá como probable respuesta un "autoritarismo" que acentuará la exclusión de las poblaciones pobres, inmigradas y minorías diversas. La otra cara posible y deseable se puede dar en las ciudades o territorios metropolitanos relativamente integrados. En ellas el conflicto se simetriza, se constituyen poderes locales fuertes y las demandas sociales pueden agregarse y llegar a

18 Delarue (1991), delegado del gobierno para la ciudad (equivale a viceministro, dependiente del jefe de gobierno), utilizó ya el concepto de "lucha de clases en el territorio". El CENSIS (Centro de Estudios Sociales, Roma) acuñó el concepto de "conflictividad asimétrica" en sus informes anuales, a principios de los ' 90.

19 Ver también su artículo "I 'senza potree' protagonisti del futuro”, en Cobelli y Grazia (2005). generar una sociedad política que exprese valores y reivindicaciones de ciudadanía. El urbanismo no garantiza la integración ciudadana plena, que depende también del empleo, el acceso a la educación y la cultura, el reconocimiento de derechos iguales para todos los habitantes, etc. Pero el urbanismo sí que crea condiciones que facilitan considerablemente la integración ciudadana, o al contrario son factores de marginación.

En los años noventa prevaleció en la cultura urbanística la "adaptación de la oferta urbana" a las nuevas condiciones de la globalización. A partir de este principio se promovieron nuevas formas de planeamiento, el estratégico especialmente. Nuevas formas de gestión: la cooperación público-privada y reformas político-administrativas: la descentralización territorial y funcional. La competitividad sustituyó a la calidad de vida. El urbanismo priorizó el proyecto sobre el plan, el proyecto arquitectónico substituyó en muchos casos al urbanístico. Y el promotor inmobiliario y el arquitecto divino impusieron con frecuencia sus intereses y sus decisiones a los responsables políticos.

Ahora, ya entrado el nuevo siglo nos parece que debemos sustituir de entrada en el lenguaje, la adaptación-sumisión a la globalización por la resistencia y las formas alternativas a los impactos negativos de la misma. Los instrumentos heredados pueden servir: estrategias y consensos, planes y proyectos, iniciativas públicas y cooperación privada, descentralización y participación ciudadana. Pero se trata de leerlos y utilizarlos a partir de objetivos integradores y sostenibles, de la reelaboración de los derechos ciudadanos y del derecho a la ciudad y de la construcción de un nuevo consenso ciudadano democrático, que no se genera sin asumir conflictos con las dinámicas disgregadoras actuantes y los actores que las promueven.

En resumen, la ciudad democrática es una conquista permanente, un campo abierto de confrontación de valores e intereses, un desafío a la innovación política, a la imaginación urbanística y a la movilización cívica.

\section{Sobre el hipotético modelo Barcelona}

A continuación exponemos una breve reflexión sobre el "modelo Barcelona", un caso en el que la 
dialéctica urbana, la confrontación en torno a las políticas urbanas y las contradicciones inherentes a las mismas se han manifestado con formas muy explícitas a lo largo de los últimos 25 años. Esta parte puede considerarse como un caso al que se aplica la reflexión general expuesta anteriormente. Utilizamos el esquema analítico desarrollado en este artículo como clave interpretativa de un proceso que se ha tendido a analizar, en la extensa bibliografía que ha producido, de forma unilateral o maniquea. Durante un largo período ha prevalecido una presentación del proceso barcelonés como un prolongado "camino de perfección", especialmente en la literatura urbanística de los años 90. En estos años de inicio del siglo XXI destaca el énfasis que ha adquirido la visión crítica, tanto en las publicaciones internacionales como en las locales.

¿Existe un modelo de transformación urbana denominable Barcelona (Borja, 1995) ${ }^{20}$ ? ¿Es solamente una marca, un label de marketing urbano exitoso (Balibrea, 2004) ${ }^{21}$ ? Existe en todo caso una percepción social (local e internacional), tanto en el ámbito político como en el intelectual, que el urbanismo barcelonés de los años ' 80 y '90 se ha caracterizado por un conjunto de políticas públicas que han configurado unas prácticas y unos discursos coherentes y que se han reflejado tanto en las formas físicas como en los usos sociales del territorio. El referirse a ello como modelo, es decir como algo ejemplar y transferible a otras ciudades, ha sido en parte una operación promocional de la ciudad (de su gobierno, de sectores profesionales y de algunas empresas de servicios). Pero la fama y la "inspiración" en Barcelona, y a veces la copia, se ha debido sobre todo a la necesidad de otras ciudades de encontrar experiencias que sirvieran

20 Marshall (2004) ha publicado un conjunto de textos que ofrecen un panorama analítico muy completo sobre el "modelo Barcelona", incluyendo una perspectiva crítica al final. Otro texto crítico más reciente sobre el urbanismo barcelonés, es el número especial de la Revista Area (2006), publicado en inglés e italiano. También, un análisis también crítico y sintético, aunque muy completo, se encuentra en la obra del geógrafo Horacio Capel (2005). Finalmente, la revista Barcelona Metrópolis Mediterránea (2007), editada por el Ayuntamiento de la Ciudad, ha publicado recientemente un Informe sobre el Modelo de Barcelona. hall (2004). de ejemplo y de legitimación ante la necesidad de inventar "nuevas políticas" que respondieran a los desafíos de la globalización. Ya es sabido que la imaginación no es la principal cualidad de las instituciones.

El "modelo" barcelonés" parte de unas premisas interesantes, aunque no es evidente que estén todavía vigentes y menos aún que lo hecho sea un modelo aplicable a otras ciudades. Siempre es posible recibir estímulos de otras experiencias: la práctica urbana avanza principalmente mediante comparaciones y confrontaciones, y se aprende de los éxitos y fracasos de los otros. Pero nunca es recomendable aplicar recetas de un caso al otro. La mediación necesaria es la que proporciona la elaboración intelectual de la cultura urbanística.

\section{Las premisas que se dan a finales de los años '70 como se verá son muy específicas}

La coyuntura política y cultural es la de los inicios de la democracia. La década que precedió a la democracia fue de gran movilización cívica, tanto en los ámbitos ciudadanos barriales como en los medios profesionales. La crítica al urbanismo desarrollista, la recuperación de lo mejor de las propuestas de Cerdà y del movimiento moderno, la elaboración de propuestas para cada barrio y para la ciudad basadas en una concepción igualitaria del espacio público y los equipamientos y la legitimación de un urbanismo participativo construyeron un consenso activo que las fuerzas políticas no podían dejar de lado $^{22}$.

En este marco ganan las primeras elecciones los partidos de izquierda, que representan la movilización cívica anterior y cuyos programas democratizadores y regeneracionistas son aceptables por parte de los sectores empresariales. Éstos precisan de unas políticas públicas que creen una oferta urbana de calidad para que invertir en la ciudad sea rentable y al mismo tiempo que se den respuestas que regulen la conflictividad social en el territorio. Esta alianza saintsimoniana (la de los "productivos" de la parábola de Saint Simón) se construye en los 80 , especialmente con la no-

22 Sobre el movimiento cívico o popular urbano ver Huertas Clavería y Andreu (1996). Ver también Borja (1987). 
minación de Barcelona como sede de los Juegos Olímpicos en 1986. La década siguiente estará marcada por los resultados de la iniciativa pública hegemónica.

El éxito es indiscutible, y sobre todo vistoso. Unos 300 proyectos realizados de espacios públicos y de equipamientos de calidad repartidos en toda la ciudad. Se proyecta una ambiciosa política de generación de nuevas centralidades, del eje del frente de mar y de la transformación del este de la ciudad (zona de industrias e infraestructuras relativamente obsoletas). Proyectos integrales de regeneración de barrios. Oferta cultural diversificada y revalorización del patrimonio arquitectónico. Descentralización hacia los distritos y los barrios y reconocimiento de los interlocutores sociales. Infraestructuras ciudadanas que comunican los fragmentos de la ciudad y de su entorno inmediato. La ciudad cambia de imagen, se reducen las desigualdades sociales en el territorio, se genera empleo, la ciudad se posiciona favorablemente en los flujos internacionales.

Pero a mediados de los '90 las condiciones iniciales habían cambiado. La ciudad se ha enriquecido y la inversión privada en la ciudad se ha hecho muy rentable. El gobierno local por el contrario se ha empobrecido, tanto en el plano económico como cultural y necesita hacer del label Barcelona un factor de atracción de capitales y turistas. Las políticas urbanas anteriores en parte se mantienen, pero en dura y casi siempre en desigual competencia con nuevas políticas más acordes con las condiciones de la globalización y del mercado.

El éxito ha tenido también efectos perversos. Los precios del suelo y de las viviendas se disparan. Los new projects (Ajuntament de Barcelona, $1995)^{23}$ es simplemente vender la ciudad al promotor privado y el resultado más emblemático es la discutible operación Diagonal Mar. La presión del sector privado conlleva la realización de enclaves, de parques temáticos, de operaciones segregadas, de destrucción del patrimonio arquitectónico (especialmente la herencia de la ciudad industrial), de deslocalización de sectores medios y bajos ha-

\footnotetext{
23 Una síntesis de este documento en Marshall
} (2004). cia la región metropolitana, de crecimiento de la urbanización difusa sin que corresponda a un crecimiento de la población (Harvey y Smith, 2005). El "modelo Barcelona" se pone en cuestión. O en todo caso se puede hablar de un "contra modelo" que entra en contradicción con el anterior.

El Forum 2004 se ha convertido en el paradigma de los críticos, tanto de los que hacen la crítica desde la defensa de lo mejor del primer modelo (Borja y Muxí, 2004) ${ }^{24}$ como los hipercríticos que consideran ambos modelos dos caras de la misma moneda (AA.VV., 2004; Delgado, 2004; 2005).

El Forum, relativo fracaso cultural (a pesar del indudable valor de los "Diálogos" en los que participaron unas 70.000 personas y 3.000 ponentes) y sobre todo político (o de marketing), es en su dimensión urbanística una expresión de la ciudad que apuesta por la inserción global: se trata de un urbanismo orientado a la demanda externa.

Mientras la ciudad-centro (Barcelona municipio) se orienta a ser un parque temático de terciario que pretende ser de "excelencia" aunque predomina la oferta de servicios de ocio, en la región metropolitana emergen las contradicciones propias de la urbanización globalizada. Espacios fragmentados por autopistas mientras que la red del "ferro" (tren, metro, tranvía) sigue con mucho retraso. Desarrollos privatizados de baja densidad de urbanizaciones de viviendas adosadas o no. Hiperconsumo de suelo, agua y servicios en general. Segregación social y funcional crecientes. Urbanización discontinua y despilfarradora. Enclaves especializados.

Pero hay otra cara de la realidad. Un territorio estructurado por ciudades medias dotadas de potencial de centralidad. Una conciencia colectiva que se expresa en demandas de calidad de vida y

24 Ver la ya citada excelente síntesis de Capel (2005), Montaner (2003) -una selección de artículos de 1984 a 2001, y Borja (1995). Sobre el modelo Barcelona es imprescindible la consulta de la colección de publicaciones de Aula Barcelona, iniciada en 1999, titulada precisamente Model Barcelona (en catalán, con un resumen en inglés). No son textos críticos, pero sí que se trata de síntesis bien informadas realizadas por los profesionales protagonistas de la gestión urbana de los años ' 80 y '90 (edición a cargo de la Fundació Bosch Gimpera-Universitat de Barcelona). Para una visión más crítica ver la excelente revista de la FAVB, El Carrer. 
desarrollo sostenible. Un tejido económico y cultural diversificado. Unas voluntades políticas, no siempre hegemónicas, que no se quieren someter a la lógica mercantil a cualquier precio. $\mathrm{Y}$ un sentido crítico en la ciudad central, en Barcelona, que cuestiona la deriva del supuesto modelo ideal que en la última década se ha sometido demasiadas veces a la contrarrevolución urbana.

\section{Referencias bibliográficas}

AA.VV. (2004). Barcelona marca registrada. Un modelo para desarmar. Barcelona: Virus Editorial.

Ajuntament de Barcelona (1988). Áreas de nuevas centralidades. Revista Barcelona Metrópolis Mediterránea, 8.

(1995). Barcelona regional: new projects. Barcelona: Ajuntament de Barcelona.

Amendola, G. (2001). Ciudad Postmoderna. Madrid: Celeste.

Angotti, T. (1993). Metropolis 2000, planning, poverty and politics. New York: Routledge.

Appadurai, A. (2001). La modernidad desbordada: dimensiones culturales de la globalización. Buenos Aires: Trilce-FCE.

(2002). Urban governamentality and the horizon of politics. Public Culture, 14.

(2007). El rechazo de las minorías. Barcelona: Tusquets.

Ascher, F. (2003). Los nuevos principios de urbanismo. Madrid: Alianza.

Balibrea, M. P. (2004). Barcelona, del modelo a la marca. Recuperado el 6 de noviembre de 2007, de www.desacuerdos.org.

Baudrillard, J. \& Nouvel, J. (2000). Les objets singuliers: architecture et philosophie. Paris: Calmann-Lévy.

Bohigas, O. (1985). Reconstrucció de Barcelona, Edicions, 62.

Bohigas, O. (2004). Contra la incontinència urbana. Reconsideració moral de l'arquitectura i la ciutat. Barcelona: Diputació de Barcelona.

Borja, J. \& Castells, M. (1997). Local y global. La gestión de las ciudades en la era de la información. Madrid: Taurus.
Borja, J. \& Muxí, Z. (Eds.) (2004). Urbanismo en el S. XXI: Bilbao, Madrid, Valencia, Barcelona. Barcelona: UPC.

Borja, J. (1987). Por unos ayuntamientos democráticos, descentralización y participación ciudadana. Madrid: IEAL.

Borja, J. (2003). La ciudad conquistada. Madrid: Alianza.

Borja, J. (2005a). El futuro urbano tiene un corazón antiguo. Catálogo de la exposición Quórum, Institut de cultura de Barcelona, Barcelona.

Borja, J. (2005b). Urbanisme i ciutadania. Revista Barcelona metrópolis mediterránea.

Borja, J. (2006). Miedos urbanos y seguridad ciudadana. Revista Catalana de Seguridad Pública.

Borja, J. (Ed.) (1995). Barcelona, un modelo de transformación urbana, 1980-1995. Quito: Programa de Gestion Urbana (PGU-LAC), Banco Mundial y NN.UU.

Borja, J. (en prensa). Gobernabilidady planeamiento de los nuevos territorios urbanos. Santiago de Chile: Instituto de Estudios Urbanos y Territoriales, Pontificia Universidad Católica.

Busquets, J. (2004). Barcelona, la construcción urbanistica de una ciudad compacta. Barcelona: Ediciones del Serbal.

Capel, H. (2005). El modelo de Barcelona, un examen crítico. Barcelona: Ediciones del Serbal.

Castells, M. \& Hall, P. (1994). Las tecnópolis del mundo. Formación de complejos industriales del siglo XXI. Madrid: Alianza.

Castells, M. (1997). La era de la información. Madrid: Alianza.

(2001). La sociología urbana de Manuel Castells. Madrid: Alianza.

Cohen, M. (1991). Política urbana y desarrollo económico: un programa para el decenio de 1990. Washington, D. C.: Banco mundial.

Cohen, M.; Blair, A. \& Tulchin, J. (Eds.) (1996). Preparing for the urban future: global pressures and local forces. Washington D. C.: Wilson Center - Smithsonian Institute.

Davis, M. (2004). Planeta de ciudades-miseria. New Left Review, 26.

\section{Verso.} (2006). Planet of slums. London:

Dear, M. (Ed.) (2002). From Chicago to Los Ángeles. London: Sage Publications. 
Delarue, J. (1991). Banlieus en difficulté: la rélégation. Paris: Cyros.

Delgado, M. (2004). La otra cara del Forum de las Culturas S.A. Barcelona: Edicions Bellaterra. (2005). Elogi del vianant, del model Barcelona a la Barcelona real. Barcelona: Edicions 1984.

Duany, A.; Plater-Zyberk, E. \& Speck, J. (2000). The rise of sprawl suburb and the decline of nation. New York: North Point Press.

Fundación Alternativas (2004). Informe sobre los derechos ciudadanos. Documento de trabajo, 51.

Gambrill, M. (2002). La globalización y sus manifestaciones en América del Norte. México, D. F.: UNAM-CISAM.

García Espuche, A. \& De Navas, T. (1999). La reconquista de Europa 1980-1999. Barcelona: CCCB.

Harvey, D. \& Smith, N. (2003). Capital financiero, propiedad inmobiliaria y cultura. Barcelona: ContraTextos, UAB-MACBA.

Harvey, D. (2005). El arte de la renta. En N. Smith \& D. Harvey (2005), Capital financiero, propiedad inmobiliaria y cultura. Barcelona: MACBA-Universidad Autónoma de Barcelona. Akal. (2005). Espacios de esperanza, Madrid:

Huertas Clavería, J. M. \& Andreu, M. (1996). Barcelona en lluita, el moviment urbà 1965-95. Barcelona: FAVB.

Ingersoll, R. (1996). Tres tesis sobre la ciudad. Revista de Occidente, 185, 11-44.

Jacobs, J. (1961). The death and life of great american cities. New York: Vintage Books USA.

(2004). Dark age ahead. New York: Ramdom House.

Kempf, H. (2005, 14 de abril). L'urbanisation grignote sans répit le territoire français. $L e$ monde.

Marshall, T. (2004). Transforming Barcelona. London: Routledge.

Masboungi, A. \& Bourdin, A. (2004). Urbanisme des modes de vie. París: Editions du Moniteur.
Masboungi, A. (1994-1997). Colección Projet Urbain. París: Ministere d'Equipement. (2001). Fabriquer la ville. Outils et methodes: les amenageurs proposent. París: La documentation française.

(2002). French urban strategies. Projets Urbains en France. París: Editions du Moniteur

Montaner, J. M. (2003). Repensar Barcelona. Barcelona: Edicions UPC.

Muñoz, F. (2004). Urbanalització: la producció residencial de baixa densitat a la provincia de Barcelona, 1985-2001. Tesis doctoral, Universitat Autonoma de Barcelona, España.

Muxí, Z. (2004). La arquitectura de la ciudad global. Barcelona: G. Gili.

Rogers, R. (2001). Towards an urban renaissance. Londres: Taylor \& Francis.

Sassen, S. (1991). The global city. New York, London, Tokio. Princeton: University Press. (2004). En Diálogos sobre la ciudad del siglo XXI. Forum de las culturas, Barcelona, España.

(2005). I "senza potree" protagonisti del futuro. En V. Cobelli \& N. Grazia (Eds.), Atlante di un'altra economia. Politiche e pratiche del cambiamento, Manifestolibri.

Sennett, R. (2003). El respeto. Barcelona: Anagrama.

Silvestre, G. (2002). Un sublime atardecer. El comercio simbólico entre arquitectos y filósofos. Punto de Vista, diciembre.

Smith, N. (2005). El redimensionamiento de las ciudades. En N. Smith \& D. Harvey (2005), Capital financiero, propiedad inmobiliaria $y$ cultura. Barcelona: MACBA-Universidad Autónoma de Barcelona.

Soja, E. (2000). Postmetropolis: critical studies of cities and regions. Oxford: Basil Blackwell.

Sorkin, M. (2005). Variaciones sobre un parque temático. Barcelona: Gustavo Gili. 